\title{
Fibrewise Decomposition of Generalized Suspension Spaces and Loop Spaces
}

By

\author{
Nobuyuki ODA*
}

\begin{abstract}
We work in the category $\operatorname{Top}_{B}^{B}$ of fibrewise pointed topological spaces over $B$. Let $l$ be a co-Hopf space (which need not be co-associative) in $\mathbf{T o p}_{B}^{B}$. The $I_{B}{ }^{\prime}$-suspension space $I_{B} X$ and the $\Gamma_{B}$-loop space $I_{B}^{*} X$ of a fibrewise pointed space $X$ over $B$ are defined as generalization of the usual suspension space $2 \mathcal{A}$ and the loop space $\Omega X$ respectively. $\Gamma_{B}$-suspension spaces and $\Gamma_{B}$-loop spaces have some properties similar to those of the usual suspension spaces and loop spaces. This is an example of Eckmann-Hilton duality. In this paper, decomposition theorems of $I_{B}{ }_{B}$-suspension space $\Gamma_{B} X$ and $\Gamma_{B}$-loop space $\Gamma_{B}^{*} X$ are proved. Short exact sequences of homotopy sets involving $I_{B}$-suspension spaces or $\Gamma_{B}$-loop spaces are obtained in the category of algebraic loops.
\end{abstract}

\section{Introduction}

The suspension space $\Sigma X$ of a topological space $X$ is defined by $\Sigma X=$ $S^{1} \wedge X$, the smash product of 1 -sphere $S^{1}$ and the space $X$. The loop space of $X$ is $\Omega X=\operatorname{map}_{*}\left(S^{1}, X\right)$, the space of base point preserving continuous maps $f: S^{1} \rightarrow X$ with compact-open topology.

Let $\Gamma$ be a co-Hopf space in $\mathbf{T o p}_{B}^{B}$, that is, a fibrewise co-Hopf space over $B$ (cf. James $[7,8]$ ). For each fibrewise pointed space $X$ over $B$, we define the $\Gamma_{B}$-suspension space $\Gamma_{B} X$ of $X$ by $\Gamma_{B} X=\Gamma \wedge_{B} X$ and the $\Gamma_{B}$-loop space $\Gamma_{B}^{*} X$ of $X$ by $\Gamma_{B}^{*} X=\operatorname{map}_{B}^{B}(\Gamma, X)$. If $B=*$ and $\Gamma=S^{1}$, then the $\Gamma_{B^{-}}$suspension space $\Gamma_{B} X$ is the usual suspension space $\Sigma X$ and the $\Gamma_{B}$-loop space $\Gamma_{B}^{*} X$ is the usual loop space $\Omega X$. If $\Gamma=\Sigma=B \times S^{1}$ of $\mathbf{T o p}_{B}^{B}$, then $\Gamma_{B} X=\Sigma_{B} X$, the reduced fibrewise suspension space of $X$ and $\Gamma_{B}^{*} X=\Omega_{B} X$, the fibrewise loop space of $X$. (We remark that James $[7,8]$ uses the symbols $\sum_{B}^{B} X$ and $\Omega_{B}^{B} X$ for reduced fibrewise suspension space and fibrewise loop space respectively. But we use our abbreviated symbols in this paper for simplicity, since we work only in the category $\operatorname{Top}_{B}^{B}$ of fibrewise pointed topological spaces over B.)

The purpose of this paper is to extend some of the familiar results on the

Communicated by K. Saito, June 7, 1993.

1991 Mathematics Subject Classifications: 55P30, 55P35, 55P40.

* Department of Applied Mathematics, Faculty of Science, Fukuoka University. 8-19-1 Nanakuma, Jonanku, Fukuoka, 814-01, Japan. 
usual suspension spaces and loop spaces to $\Gamma_{B^{-}}$-suspension spaces and $\Gamma_{B^{-}}$-loop spaces in $\operatorname{Top}_{B}^{B}$.

In $\S 1$ we review some definitions in $\operatorname{Top}_{B}^{B}$ and prove fundamental results on decompositions of co-Hopf spaces and Hopf spaces in Top $\mathbf{T}_{B}^{B}$. Hilton, Mislin and Roitberg [5] obtained a decomposition theorem of co-Hopf spaces under some conditions in Theorem 4.2 of [5]. The result of (2) of the following Theorem 1.1 is a generalization of their result. We call a co-Hopf space $A$ in $\operatorname{Top}_{B}^{B}$ a co-looplike space in $\operatorname{Top}_{B}^{B}$ or a fibrewise co-looplike space over $B$ if $[A, Z]_{B}^{B}$ is naturally an algebraic loop for each space $Z$ in $\mathbf{T o p}_{B}^{B}$ (cf. $\S 1$ ).

Theorem 1.1. Let $A \stackrel{\imath}{\rightarrow} X \stackrel{q}{\rightarrow} C$ be a fibrewise pointed cofibration sequence of fibrewise co-looplike spaces and fibrewise co-Hopf maps with fibrewise pointed homotopy retraction $r: X \rightarrow A$ such that $r \circ i \simeq{ }_{B} 1_{A}$ ( $r$ need not be a fibrewise coHopf map), then the following results hold.

(1) There is a short exact sequence

$$
0 \longrightarrow[C, Z]_{B}^{B} \stackrel{q^{*}}{\longrightarrow}[X, Z]_{B}^{B} \stackrel{i^{*}}{\longrightarrow}[A, Z]_{B}^{B} \longrightarrow 0
$$

of algebraic loops and homomorphisms for any space $Z$ in $\mathbf{T o p}_{B}^{B}$.

(2) There is a fibrewise pointed homotopy decomposition

$$
X \simeq{ }_{B} C \vee{ }_{B} A .
$$

We also prove the dual result of the above theorem. In $\S 2$ we study $\Gamma_{B^{-}}$ suspension space for any fibrewise co-looplike space $\Gamma$ over $B$. By Theorem 1.1 above we have, for example, the following result (Theorem 2.1(2));

Let $i: A \subset X$ be a cofibration in $\mathbf{T o p}_{B}^{B}$. If $A$ is $\Gamma_{B}$-retractile in $X$ (that is, there exists a fibrewise map $r: \Gamma_{B} X \rightarrow \Gamma_{B} A$ such that $r \Gamma_{B} i \simeq_{B} 1_{\Gamma_{B} A}$ ), then we have a fibrewise pointed homotopy decomposition

$$
\Gamma_{B} X \simeq_{B} \Gamma_{B}\left(X /{ }_{B} A\right) \vee{ }_{B} \Gamma_{B} A .
$$

When $B=*$ and $\Gamma=S^{1}$, Theorem 2.1(2) mentioned above is a well-known result (cf. (15.1) of Baues [1] and (6.27) of James [7]). For $\Sigma=B \times S^{1}$, see 4 (p. 175) of James [8]. This enables us to prove, for example, the following decompositions.

Theorem 2.2. Let $\Gamma$ be a fibrewise co-looplike space. Let $X$ and $Y$ be fibrewise non-degenerate spaces. Let $M$ be any subspaces of $X \times{ }_{B} Y$ such that

$$
X \vee{ }_{B} Y \subset M \subset X \times{ }_{B} Y,
$$

and $\bar{\jmath}: X \vee_{B} Y \rightarrow M$ a fibrewise pointed cofibration. Then we have a fibrewise pointed homotopy decomposition

$$
\Gamma_{B} M \simeq_{B} \Gamma_{B} X \vee_{B} \Gamma_{B} Y \vee_{B} \Gamma_{B}\left\{M /{ }_{B}\left(X \vee_{B} Y\right)\right\}
$$


To prove decompositions of subspaces of fibrewise product space $X \times_{B} Y$ in Theorems 2.2, 2.4, 2.5 and Corollary 2.3, the concept fibrewise non-degenerate space of James [8] plays a very important role.

The special cases of Theorem 2.2 and Corollary 2.3 are known when $B=*$ and $\Gamma=\Sigma=S^{1}$ (cf. (15.9) of Baues [1] and Lemma 1.1.5.1 of Zabrodsky [14]). For the case $\Sigma=B \times S^{1}$ in $\mathbf{T o p}_{B}^{B}$, see (22.6) of James [8].

In $\S 3$, we study some properties of $\Gamma_{B}$-loop spaces. We prove dual formulas of those in $\S 2$. We may consider these results as Eckmann-Hilton duality (cf. $\S 11$ of Hilton [4]). The $\Gamma_{B^{-}}$suspension functor and the $\Gamma_{B}$-loop functor are different from the suspension functor and the loop functor in algebraic homotopy theory (cf. Baues [1] or Quillen [10]). Let $\Sigma=B \times S^{1}$. In $\S 4$ we show that $\Sigma_{B}$-retractile implies $\Gamma_{B}$-retractile and $\Sigma_{B}^{*}$-retractile implies $\Gamma_{B}^{*}$-retractile for any co-Hopf space $\Gamma$ in $\mathbf{T o p}_{B}^{B}$. The author would like to thank Professor $\mathrm{N}$. Iwase for suggesting that retractile implies $\Gamma$-retractile when the co-Hopf space $\Gamma$ is a CW-complex in the category of topological spaces with base point.

\section{$\S 1$. Decompositions of Fibrewise Spaces over $B$}

Let Top be the category of topological spaces. We define the category $\operatorname{Top}_{B}^{B}$ of the fibrewise pointed topological spaces over $B$ following James [7] and $[8]$.

An object in $\operatorname{Top}_{B}^{B}$ is a pair of maps $B \stackrel{s}{\rightarrow} X \stackrel{p}{\rightarrow} B$ in Top such that $p \circ s=1_{B}$, the identity map. For each point $b \in B$, we regard $s(b)$ the base point of the subspace $p^{-1}(b)$, the fibre over $b$. We write $s(b)=*_{b}$ and call $*_{B}=\left\{*_{b} \mid b \in B\right\}$ the fibrewise base point.

A morphism $f:(B \stackrel{s}{\rightarrow} X \stackrel{p}{\rightarrow} B) \rightarrow(B \stackrel{t}{\rightarrow} Y \stackrel{q}{\rightarrow} B)$ in $\mathbf{T o p}_{B}^{B}$ is a map $f: X \rightarrow Y$ in Top such that $f \circ s=t$ and $q \circ f=p$. We write $f: X \rightarrow \vec{B} Y$ or simply $f: X \rightarrow Y$ for a morphism in $\mathbf{T o p}_{B}^{B}$.

Thus $\operatorname{Top}_{B}^{B}$ is a category of fibrewise pointed topological spaces and fibrewise pointed maps. A fibrewise pointed homotopy relation is denoted by $\simeq_{B}$ and the set of the fibrewise pointed homotopy classes in $\mathbf{T o p}_{B}^{B}$ is denoted by $[X, Y]_{B}^{B}$.

The fibrewise wedge sum $X \vee_{B} Y$ is a subspace of fibrewise product $X \times_{B} Y$ by the inclusion map $j_{B}: X \vee_{B} Y \subset X \times_{B} Y$. We denote by $\Delta_{X, B}: X \rightarrow X \times_{B} X$ the fibrewise diagonal map and $\nabla_{X, B}: X \vee_{B} X \rightarrow X$ the fibrewise folding map. We denote by $*_{B}: X \rightarrow Y$ the fibrewise constant map.

Let $A$ be a subspace of $X$ in $\mathbf{T o p}_{B}^{B}$. Then the fibrewise quotient space is denoted by $X /{ }_{B} A$. The fibrewise smash product is defined by $X \wedge{ }_{B} Y=$ $\left(X \times{ }_{B} Y\right) /{ }_{B}\left(X \vee_{B} Y\right)$. The fibrewise pointed mapping-space ( 99 of [8]) is denoted by $\operatorname{map}_{B}^{B}(Y, Z)$ and we have an isomorphism of fibrewise homotopy sets

$$
\left[X \wedge{ }_{B} Y, Z\right]_{B}^{B} \cong\left[X, \operatorname{map}_{B}^{B}(Y, Z)\right]_{B}^{B}
$$

(cf. (9.14) and (9.25) of [8]). Let $\Sigma=B \times S^{1}$ in $\operatorname{Top}_{B}^{B}$. Then $\Sigma_{B} X=\Sigma \wedge_{B} X$ is 
the fibrewise reduced suspension space of $X$ and $\Omega_{B} X=\sum_{B}^{*} X=\operatorname{map}_{B}^{B}(\Sigma, X)$ is the fibrewise loop space of $X$.

Let $S$ be a set with a binary operation + (not necessarily commutative nor associative here). We call $S$ an algebraic loop if $S$ has two-sided identity (denoted by 0 ) and for any elements $a, b$ of $S$, the equations

$$
x+a=b \text { and } a+y=b
$$

have a unique pair of solutions $x, y \in S$ (cf. James [6], Rutter [11], Hilton, Mislin and Roitberg [5]).

A map $\tau: S \rightarrow L$ between two algebraic loops is called a homomorphism if $\tau(a+b)=\tau(a)+\tau(b)$ holds for any $a, b \in S$. If $\tau: S \rightarrow L$ is a homomorphism, we have $\tau(0)=0$. A sequence

$$
S \stackrel{\tau}{\longrightarrow} L \stackrel{\sigma}{\longrightarrow} R
$$

of algebraic loops and homomorphisms is said to be exact if $\operatorname{Im} \tau=\operatorname{Ker} \sigma$. Let us suppose that the sequence (1) is exact and $\sigma(b)=\sigma(c)$ for elements $b, c \in L$. Since $L$ is an algebraic loop, there exist unique elements, $d, d^{\prime} \in L$ such that $d+b=c$ and $b+d^{\prime}=c$. Since $\sigma$ is a homomorphism and $R$ is an algebraic loop, we have $\sigma(d)=\sigma\left(d^{\prime}\right)=0$. Then there exist elements $a, a^{\prime} \in S$ such that $\tau(a)=d$ and $\tau\left(a^{\prime}\right)=d^{\prime}$. Thus we have shown that if $\sigma(b)=\sigma(c)$, then there exist $a, a^{\prime}$ such that $\tau(a)+b=c$ and $b+\tau\left(a^{\prime}\right)=c$. Especially, if $S=0$ then $\sigma$ is a monomorphism.

By the above argument, we can use the terminology "long exact sequence" and "short exact sequence" in the category of algebraic loops and homomorphisms. (cf. $§ 1.3$ of Zabrodsky [14]).

A co-Hopf space $A$ in $\mathbf{T o p}_{B}^{B}$ or a fibrewise co-Hopf space $A$ over $B$ is a space with a fibrewise co-multiplication $\theta: A \rightarrow A \vee_{B} A$, that is, the relation $j_{B^{\circ}} \theta \simeq{ }_{B} \Delta_{A, B}$ holds for the inclusion map $j_{B}: A \vee{ }_{B} A \rightarrow A \times{ }_{B} A$ and the fibrewise diagonal map $\Delta_{A, B}: A \rightarrow A \times_{B} A$ (cf. $\S 19$ of [8]).

Let $A$ be a co-Hopf space in $\operatorname{Top}_{B}^{B}$ with a fibrewise co-multiplication $\theta: A \rightarrow$ $A \vee_{B} A$. For any maps $\alpha, \beta: A \rightarrow Z$ in $\operatorname{Top}_{B}^{B}$, we define a map $\alpha \dot{+}_{B} \beta: A \rightarrow Z$ in $\operatorname{Top}_{B}^{B}$ by

$$
\alpha \dot{+}_{B} \beta=\nabla_{Z, B^{\circ}}\left(\alpha \vee_{B} \beta\right) \circ \theta,
$$

where $\nabla_{Z, B}: Z \vee_{B} Z \rightarrow Z$ is the fibrewise folding map (cf. Oda [9]).

A co-looplike space $A$ in $\operatorname{Top}_{B}^{B}$ or a fibrewise co-looplike space $A$ over $B$ is a fibrewise co-Hopf space over $B$ which induces an algebraic loop structure in $[A, Z]_{B}^{B}$ with the binary operation $\dot{+}_{B}$ for any space $Z$ in $\mathbf{T o p}_{B}^{B}$. A fibrewise co-Hopf map $f: A \rightarrow A^{\prime}$ between fibrewise co-Hopf spaces $(A, \theta)$ and $\left(A^{\prime}, \theta^{\prime}\right)$ is a fibrewise pointed map which makes the diagram 


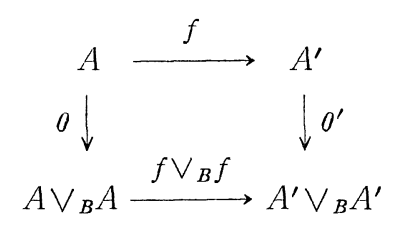

fibrewise pointed homotopy commutative.

An inclusion map $i: A \subset X$ is called a cofibration in $\mathbf{T o p}_{B}^{B}$ or fibrewise pointed cofibration if it has the fibrewise pointed homotopy extension property (cf. $\S 21$ of [8]).

Theorem 1.1. Let $A \stackrel{i}{\rightarrow} X \stackrel{q}{\rightarrow} C$ be a fibrewise pointed cofibration sequence of fibrewise co-looplike spaces and fibrewise co-Hopf maps with fibrewise pointed homotopy retraction $r: X \rightarrow A$ such that $r \circ i \simeq{ }_{B} 1_{A}$ ( $r$ need not be a fibrewise coHopf map), then the following results hold.

(1) There is a short exact sequence

$$
0 \longrightarrow[C, Z]_{B}^{B} \stackrel{q^{*}}{\longrightarrow}[X, Z]_{B}^{B} \stackrel{i^{*}}{\longrightarrow}[A, Z]_{B}^{B} \longrightarrow 0
$$

of algebraic loops and homomorphisms for any space $Z$ in $\mathbf{T o p}_{B}^{B}$.

(2) There is a fibrewise pointed homotopy decomposition

$$
X \simeq{ }_{B} C \vee{ }_{B} A .
$$

(It does not preserve fibrewise co-Hopf structure in general.)

Proof. (1) Consider a fibrewise pointed cofibration sequence

$$
A \stackrel{i}{\longrightarrow} X \stackrel{q}{\longrightarrow} C \stackrel{\stackrel{o}{\longrightarrow}}{\longrightarrow} \Sigma_{B} A \stackrel{\Sigma_{B} i}{\longrightarrow} \Sigma_{B} X \longrightarrow \cdots
$$

(cf. $\S 21$ of [8]). Since there exists a fibrewise pointed homotopy retraction $r: X \rightarrow A$ such that $r \circ i \simeq{ }_{B} 1_{A}$ and hence also $\Sigma_{B} r \circ \Sigma_{B} i \simeq{ }_{B} 1_{\Sigma_{B} A}$, we have a short exact sequence of algebraic loops and homomorphisms

$$
0 \longrightarrow[C, Z]_{B}^{B} \stackrel{q^{*}}{\longrightarrow}[X, Z]_{B}^{B} \stackrel{i^{*}}{\longrightarrow}[A, Z]_{B}^{B} \longrightarrow 0
$$

for any space $Z$ in $\operatorname{Top}_{B}^{B}$ by a long fibrewise homotopy exact sequence.

(2) (cf. Proof of Theorem 4.2 of [5]) Let $i_{1}: C \rightarrow C \vee{ }_{B} A$ and $i_{2}: A \rightarrow C \vee_{B} A$ be the inclusion maps. Now the maps

$$
i_{1} \circ q: X \longrightarrow C \longrightarrow C \vee_{B} A \text { and } i_{2} \circ r: X \longrightarrow A \longrightarrow C \vee_{B} A
$$

define a map $\xi=\left(i_{1} \circ q\right) \dot{+}_{B}\left(i_{2} \circ r\right): X \rightarrow C \vee_{B} A$. We show that this map $\xi$ is a fibrewise pointed homotopy equivalence.

Consider the following commutative diagram 


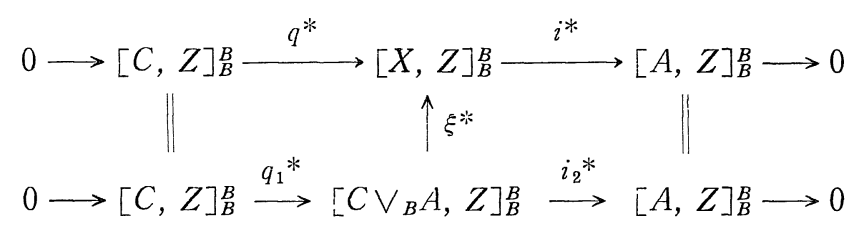

where $q_{1}: C \vee{ }_{B} A \rightarrow C$ is a projection. The horizontal sequences are exact in the category of algebraic loops and homomorphisms. (We remark that $\xi^{*}$ is not a homomorphism of loops.) Since there is an isomorphism of sets $\left[C \vee_{B} A, Z\right]_{B}^{B}$ $\cong[C, Z]_{B}^{B} \times[A, Z]_{B}^{B}$ (cf. $\S 5$ of [7] and $\S 19$ of [8]), any element of $\left[C \vee_{B} A, Z\right]_{B}^{B}$ can be written as $\langle c, a\rangle$ for unique elements $c \in[C, Z]_{B}^{B}$ and $a \in[A, Z]_{B}^{B}$. We see

$$
\begin{aligned}
\xi^{*}(\langle c, a\rangle) & =\langle c, a\rangle \circ\left\{\left(i_{1} \circ q\right) \dot{+}_{B}\left(i_{2} \circ r\right)\right\} \\
& =\left(\langle c, a\rangle \circ i_{1} \circ q\right) \dot{+}_{B}\left(\langle c, a\rangle \circ i_{2} \circ \gamma\right) \\
& =(c \circ q) \dot{+}_{B}(a \circ r) .
\end{aligned}
$$

Then using the properties of short exact sequence of algebraic loops and homomorphisms, we see that

$$
\xi^{*}:\left[C \vee_{B} A, Z\right]_{B}^{B} \longrightarrow[X, Z]_{B}^{B}
$$

is an isomorphism of sets for any space $Z$ in $\operatorname{Top}_{B}^{B}$.

We set $Z=X$ in (2). Then we have a map $\eta: C \vee_{B} A \rightarrow X$ such that

We see that

$$
\xi^{*}(\eta)=\eta \circ \xi \simeq{ }_{B} 1_{X} .
$$

$$
\eta^{*}:[X, Z]_{B}^{B} \longrightarrow\left[C \vee_{B} A, Z\right]_{B}^{B}
$$

is also an isomorphism for any space $Z$ in $\operatorname{Top}_{B}^{B}$. We set $Z=C \vee_{B} A$ in (4), then we have a map $\xi^{\prime}: X \rightarrow C \vee_{B} A$ such that

$$
\eta^{*}\left(\xi^{\prime}\right)=\xi^{\prime} \circ \eta \simeq{ }_{B} 1_{C \vee_{B} A} .
$$

Since $\eta \circ \xi \simeq{ }_{B} 1_{X}$ by (3) and $\xi^{\prime} \circ \eta \simeq{ }_{B} 1_{C \vee_{B} A}$ by (5), we have $\xi^{\prime} \simeq{ }_{B} \xi$ and hence $\xi$ is the desired fibrewise homotopy equivalence.

q.e.d.

A Hopf space $Z$ in $\mathbf{T o p}_{B}^{B}$ or a fibrewise Hopf space $Z$ over $B$ is a space with a fibrewise multiplication $\mu: Z \times_{B} Z \rightarrow Z$ such that $\mu \circ j_{B} \simeq{ }_{B} \nabla_{Z, B}$ (cf. (19.1) of [8]).

Let $Z$ be a Hopf space in $\operatorname{Top}_{B}^{B}$ with a fibrewise multiplication $\mu: Z \times{ }_{B} Z$ $\rightarrow Z$. For any maps $\alpha, \beta: X \rightarrow Z$ in $\operatorname{Top}_{B}^{B}$, we define a map $\alpha+{ }_{B} \beta: X \rightarrow Z$ in $\operatorname{Top}_{B}^{B}$ by

$$
\alpha+{ }_{B} \beta=\mu \circ\left(\alpha \times{ }_{B} \beta\right) \circ \Delta_{X, B}
$$

where $\Delta_{X, B}: X \rightarrow X \times{ }_{B} X$ is a fibrewise diagonal map (cf. [9]).

A looplike space $Z$ in $\operatorname{Top}_{B}^{B}$ or a fibrewise looplike space $Z$ over $B$ is a fibre- 
wise Hopf space over $B$ which induces an algebraic loop structure in $[A, Z]_{B}^{B}$ with the binary operation $+_{B}$ for any space $A$ in $\mathbf{T o p}_{B}^{B}$.

Theorem 1.2. Let $F \stackrel{2}{\rightarrow} E \stackrel{p}{\rightarrow} Z$ be a fibrewise pointed fibration sequence of fibrewise looplike spaces and fibrewise Hopf maps. If $p: E \rightarrow Z$ has a fibrewise pointed homotopy cross-section $s: Z \rightarrow E$ such that $p \circ s \simeq_{B} 1_{Z}$ (s need not be a fibrewise Hopf map), then the following results hold.

(1) There is a short exact sequence

$$
0 \longrightarrow[A, F]_{B}^{B} \stackrel{i_{*}}{\longrightarrow}[A, E]_{B}^{B} \stackrel{p_{*}}{\longrightarrow}[A, Z]_{B}^{B} \longrightarrow 0
$$

of algebraic loops and homomorphisms for any space $A$ in $\mathbf{T o p}_{B}^{B}$.

(2) There is fibrewise pointed homotopy decomposition

$$
E \simeq{ }_{B} F \times{ }_{B} Z .
$$

(It does not preserve fibrewise Hopf structure in general.)

Proof. (1) Since $F \stackrel{2}{\rightarrow} E \stackrel{p}{\rightarrow} Z$ is a fibrewise pointed fibration sequence, we have a long fibrewise pointed fibration sequence

$$
\cdots \longrightarrow \Omega_{B} E \stackrel{\Omega_{B} D}{\longrightarrow} \Omega_{B} Z \stackrel{\delta}{\longrightarrow} F \stackrel{i}{\longrightarrow} E \stackrel{p}{\longrightarrow} Z
$$

(cf. Crabb and James [2]). Since $p: E \rightarrow Z$ has a fibrewise pointed homotopy cross-section $s: Z \rightarrow E$ such that $p \circ s \simeq{ }_{B} 1_{Z}$ by our assumption, we have the result by a long fibrewise homotopy exact sequence.

(2) We define $\zeta: F \times{ }_{B} Z \rightarrow E$ by

$$
\zeta=\left(i \circ p_{1}\right)+{ }_{B}\left(s \circ p_{2}\right),
$$

where $p_{1}: F \times{ }_{B} Z \rightarrow F$ and $p_{2}: F \times{ }_{B} Z \rightarrow Z$ are projections. Then the result follows by a dual argument of the proof of (2) of Theorem 1.1. q.e.d.

\section{$\S 2 . \quad \Gamma_{B}$-Suspension Space}

In the following sections we assume that each fibrewise pointed topological space has closed section so that we have natural equivalence

$$
\left(X \vee_{B} Y\right) \wedge{ }_{B} Z \cong{ }_{B}\left(X \wedge_{B} Z\right) \vee_{B}\left(Y \wedge_{B} Z\right)
$$

(cf. (3.80) of [7] and (6.1) of [8]).

Let $\Gamma$ be a fibrewise co-Hopf space over $B$ with a fibrewise co-multiplication $\gamma: \Gamma \rightarrow \Gamma \vee_{B} \Gamma$ through $\S \S 2$ and 3 . We assume that $\Gamma$ is fibrewise locally compact and fibrewise regular so that we have $\Sigma_{B} \Gamma_{B} X \cong_{B} \Gamma_{B} \Sigma_{B} X$ for any space $X$ in $\operatorname{Top}_{B}^{B}$ (cf. (6.2) of [8]). We do not assume that $\Gamma$ is co-associative (=fibrewise homotopy associative $[7,8])$. For any space $X$ in Top T $_{l j}^{B}$, we define 


$$
\left.\Gamma_{B} X=\Gamma \wedge_{B} X \quad \text { (the } \Gamma_{B^{-}} \text {suspension space of } X\right) .
$$

A map $f: X \rightarrow Y$ in $\operatorname{Top}_{B}^{B}$ induces a $\Gamma_{B}$-suspension map $\Gamma_{B} f: \Gamma_{B} X \rightarrow \Gamma_{B} Y$. We see $\Gamma_{B} g \circ \Gamma_{B} f=\Gamma_{B}(g \circ f)$ for any maps $f: X \rightarrow Y$ and $g: Y \rightarrow Z$ in $\mathbf{T o p}_{B}^{B}$. We define $\gamma_{X}: \Gamma_{B} X \rightarrow \Gamma_{B} X \vee_{B} \Gamma_{B} X$ by

$$
\gamma_{X}=\gamma \wedge_{B} 1_{X}: \Gamma \wedge_{B} X \longrightarrow\left(\Gamma \vee_{B} \Gamma\right) \wedge_{B} X \cong_{B}\left(\Gamma \wedge_{B} X\right) \vee_{B}\left(\Gamma \wedge_{B} X\right)
$$

Then $\Gamma_{B} X$ is a fibrewise co-Hopf space with a fibrewise co-multiplication $\gamma_{X}$ for any fibrewise pointed space $X$ over $B$. We have formulas

$$
\alpha \circ\left(\beta \dot{+}_{B} \gamma\right)=(\alpha \circ \beta) \dot{+}_{B}(\alpha \circ \gamma) \text { and }\left(\beta \dot{+}_{B} \gamma\right) \circ \Gamma_{B} \delta=\left(\beta \circ \Gamma_{B} \delta\right) \dot{+}_{B}\left(\gamma \circ \Gamma_{B} \delta\right)
$$

for any maps $\alpha: Y \rightarrow Z, \beta, \gamma: \Gamma_{B} X \rightarrow Y$ and $\delta: W \rightarrow X$ in $\mathbf{T o p}_{B}^{B}$.

Remark. We assume that $\Gamma$ is a fibrewise co-looplike space in many statements in the following discussion. But, the assumption that $\Gamma$ is a fibrewise co-looplike space can be replaced by the assumption that each homotopy set $\left[\Gamma_{B} X, Y\right]_{B}^{B}\left(\cong\left[X, \Gamma_{B}^{*} Y\right]_{B}^{B}\right)$ which appears in our discussion is an algebraic loop with the "addition" induced by the fibrewise co-Hopf structure of $\Gamma_{B} X$ (or the fibrewise Hopf structure of $\Gamma_{B}^{*} Y$, cf. §3). Let, for example, $B=*$. If $\Gamma$ is a co-Hopf space and if $\Gamma$ and $X$ have homotopy type of connected CW-complex, then $\Gamma_{B} X$ is a co-looplike space by Saito [12] (cf. Rutter [11], Hilton, Mislin and Roitberg [5]).

Let $A$ be a subspace of $X$ in $\mathbf{T o p}_{B}^{B}$ with an inclusion map $i: A \subset X$. We say that $A$ is $\Gamma_{B}$-retractile in $X$ (or $i: A \rightarrow X$ is $\Gamma_{B}$-retractile) when there exists a fibrewise pointed map $r: \Gamma_{B} X \rightarrow \Gamma_{B} A$ such that $r \circ \Gamma_{B} i \simeq_{B} 1_{\Gamma_{B} A}$. If $A$ is a fibrewise pointed homotopy retraction of $X$, that is, there exists a fibrewise pointed map $r: X \rightarrow A$ such that $r \circ i \simeq_{B} 1_{A}$, then $A$ is $\Gamma_{B}$-retractile in $X$ for any fibrewise co-Hopf space $\Gamma$. We remark that when $B=*$ and $\Gamma=\Sigma=S^{1}$ (1-sphere), a subspace $A$ of $X$ is usually said to be retractile in $X$ if $\sum i: \Sigma A \rightarrow \Sigma X$ has a homotopy retraction $r: \Sigma X \rightarrow \Sigma A$, namely, $r \circ \sum i \simeq 1_{\Sigma A}$ (cf. $\S 3$ of [6] and (6.26) of [7]).

Theorem 2.1. Let $\Gamma$ be a fibrewise co-looplike space. Let $i: A \subset X$ be $a$ fibrewise pointed cofibration in $\mathbf{T o p}_{B}^{B}$. If $A$ is $\Gamma_{B^{-}}$retractile in $X$, then the following results hold.

(1) There is a short exact sequence

$$
0 \longrightarrow\left[\Gamma_{B}\left(X /{ }_{B} A\right), Z\right]_{B}^{B} \stackrel{\left(\Gamma_{B} q\right)^{*}}{\longrightarrow}\left[\Gamma_{B} X, Z\right]_{B}^{B} \stackrel{\left(\Gamma_{B} i\right)^{*}}{\longrightarrow}\left[\Gamma_{B} A, Z\right]_{B}^{B} \longrightarrow 0
$$

of algebraic loops and homomorphisms for any space $Z$ in $\mathbf{T o p}_{B}^{B}$.

(2) There is a fibrewise pointed homotopy decomposition

$$
\Gamma_{B} X \simeq{ }_{B} \Gamma_{B}\left(X /{ }_{B} A\right) \vee{ }_{B} \Gamma_{B} A .
$$


(It does not preserve fibrewise co-Hopf structure in general.)

Proof. Consider a long fibrewise pointed cofibration sequence

$$
A \stackrel{i}{\longrightarrow} X \stackrel{q}{\longrightarrow} X /{ }_{B} A \stackrel{\delta}{\longrightarrow} \Sigma_{B} A \stackrel{\Sigma_{B} i}{\longrightarrow} \Sigma_{B} X \longrightarrow \cdots .
$$

(cf. $\S 21$ of [8].) This implies a long fibrewise pointed cofibration sequence

$$
\Gamma_{B} A \stackrel{\Gamma_{B} i}{\longrightarrow} \Gamma_{B} X \stackrel{I^{\prime}{ }_{B} q}{\longrightarrow} \Gamma_{B}\left(X /{ }_{B} A\right) \stackrel{\Gamma_{B} \delta}{\longrightarrow} \Sigma_{B} \Gamma_{B} A \stackrel{\Sigma_{B} \Gamma_{B} i}{\longrightarrow} \Sigma_{B} \Gamma_{B} X \longrightarrow \cdots
$$

Then the result follows by Theorem 1.1, since there exists a fibrewise homotopy retraction $r: \Gamma_{B} X \rightarrow \Gamma_{B} A$ such that $r \circ \Gamma_{B} i \simeq{ }_{B} 1_{\Gamma_{B} A}$ by our assumption. q.e.d.

James studied fibrewise non-degenerate space and fibrewise well-pointed space in $\S 22$ of [8].

Theorem 2.2. Let $\Gamma$ be a fibrewise co-looplike space. Let $X$ or $Y$ be $a$ fibrewise non-degenerate space. Let $M$ be any subspace of $X \times_{B} Y$ such that

$$
X \vee_{B} Y \subset M \subset X \times{ }_{B} Y,
$$

and $\bar{\jmath}: X \vee_{B} Y \rightarrow M$ a fibrewise pointed cofibration. Then we have a fibrewise pointed homotopy decomposition

$$
\Gamma_{B} M \simeq_{B} \Gamma_{B} X \vee_{B} \Gamma_{B} Y \vee_{B} \Gamma_{B}\left\{M /{ }_{B}\left(X \vee_{B} Y\right)\right\}
$$

Proof. We assume that $Y$ is a fibrewise non-degenerate space. (The case that $X$ is a fibrewise non-degenerate space is proved similarly.) Since $X=$ $X \times{ }_{B}\left\{*_{B}\right\}$ is a fibrewise retraction of $M$ and $X \times_{B}\left\{*_{B}\right\} \rightarrow M$ is a fibrewise pointed cofibration by (21.2) of [8], we have

$$
\Gamma_{B} M \simeq_{B} \Gamma_{B} X \vee_{B} \Gamma_{B}\left\{M /{ }_{B}\left(X \times_{B}\left\{*_{B}\right\}\right)\right\}
$$

by Theorem 2.1. Let $N=M /{ }_{B}\left(X \times_{B}\left\{*_{B}\right\}\right)$. We remark that $\left\{*_{B}\right\} \times_{B} Y \rightarrow N$ is a fibrewise pointed cofibration by (21.2) of [8], for $\bar{\jmath}: X \vee_{B} Y \rightarrow M$ is a fibrewise pointed cofibration by our assumption. Since $Y=\left\{*_{B}\right\} \times_{B} Y$ is a fibrewise retraction of $N$, and

$$
N /{ }_{B} Y \cong{ }_{B}\left\{M /{ }_{B}\left(X \times{ }_{B}\left\{*_{B}\right\}\right)\right\} /{ }_{B}\left(\left\{*_{B}\right\} \times{ }_{B} Y\right) \cong{ }_{B} M /{ }_{B}\left(X \vee{ }_{B} Y\right),
$$

we have $\Gamma_{B} N=\Gamma_{B} Y \vee_{B} \Gamma_{B}\left(N /{ }_{B} Y\right)=\Gamma_{B} Y \vee_{B} \Gamma_{B}\left\{M /{ }_{B}\left(X \vee_{B} Y\right)\right\}$ by Theorem 2.1 . Thus we have $\Gamma_{B} M \simeq_{B} \Gamma_{B} X \vee_{B} \Gamma_{B} Y \vee_{B} \Gamma_{B}\left\{M /{ }_{B}\left(X \vee_{B} Y\right)\right\}$. q.e.d.

As special cases of Theorem 2.2, we have the following results.

Corollary 2.3. Let $\Gamma$ be a fibrewise co-looplike space. Let $(X, A)$ and $(Y, D)$ be any pairs of fibrewise non-degenerate spaces. Then we have the following fibrewise pointed homotopy decompositions. 
(1) $\Gamma_{B}\left(X \times_{B} Y\right) \simeq{ }_{B} \Gamma_{B} X \vee_{B} \Gamma_{B} Y^{\sim} \vee_{B} \Gamma_{B}\left(X \wedge_{B} Y\right)$

(2) $\Gamma_{B}\left\{\left(X \times_{B}\left\{*_{B}\right\}\right) \cup\left(A \times_{B} Y\right)\right\} \simeq{ }_{B} \Gamma_{B} X \vee_{B} \Gamma_{B} Y \vee_{B} \Gamma_{B}\left(A \wedge{ }_{B} Y^{\top}\right)$.

(3) $\Gamma_{B}\left\{\left(X \vee_{B} Y\right) \cup\left(A \times_{B} D\right)\right\} \simeq{ }_{B} \Gamma_{B} X \vee_{B} \Gamma_{B} Y \vee_{B} \Gamma_{B}\left(A \wedge{ }_{B} D\right)$.

Proof. Since (1) $\left(X \times_{B} Y, X \vee_{B} Y\right),(2)\left(\left(X \times_{B}\left\{*_{B}\right\}\right) \cup\left(A \times_{B} Y\right), X \vee_{B} Y\right)$ and (3) $\left(\left(X \vee_{B} Y\right) \cup\left(A \times_{B} D\right), X \vee_{B} Y\right)$ are fibrewise pointed cofibred pair by (22.7) and (21.2) of [8], we have the result by Theorem 2.2 .

$q . e . d$.

Theorem 2.4. Let $\Gamma$ be a fibrewise co-looplike space. Let $(X, A)$ and $(Y, D)$ be fibrewise pointed cofibred pairs of fibrewise non-degenerate spaces.

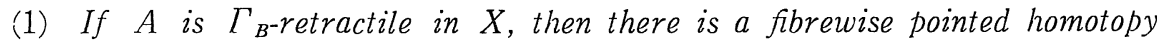
decomposition

$\Gamma_{B}\left(X \times_{B} Y\right) \simeq{ }_{B} \Gamma_{B}\left(X /{ }_{B} A\right) \vee{ }_{B} \Gamma_{B} A \vee_{B} \Gamma_{B} Y \vee_{B} \Gamma_{B}\left\{\left(X /{ }_{B} A\right) \wedge{ }_{B} Y\right\} \vee_{B} \Gamma_{B}\left(A \wedge{ }_{B} Y\right)$.

(2) If $A$ is $\Gamma_{B}$-retractile in $X$ and $D$ is $\Gamma_{B}$-retractile in $Y$, then there is a fibrewise pointed homotopy decomposition

$$
\begin{aligned}
\Gamma_{B}\left(X \times_{B} Y\right) \simeq & { }_{B} \Gamma_{B}\left(X /{ }_{B} A\right) \vee{ }_{B} \Gamma_{B} A \vee_{B} \Gamma_{B}\left(Y /{ }_{B} D\right) \vee_{B} \Gamma_{B} D \\
& \vee_{B} \Gamma_{B}\left\{\left(X /{ }_{B} A\right) \wedge{ }_{B}\left(Y /{ }_{B} D\right)\right\} \vee_{B} \Gamma_{B}\left\{\left(X /{ }_{B} A\right) \wedge{ }_{B} D\right\} \\
& \vee_{B} \Gamma_{B}\left\{A \wedge_{B}\left(Y /{ }_{B} D\right)\right\} \vee_{B} \Gamma_{B}\left(A \wedge{ }_{B} D\right) .
\end{aligned}
$$

Proof. (1) $\Gamma_{B}\left(X \times_{B} Y\right) \simeq{ }_{B} \Gamma_{B} X \vee_{B} \Gamma_{B} Y \vee_{B} \Gamma_{B}\left(X \wedge_{B} Y\right)$

$$
\begin{aligned}
& \simeq{ }_{B} \Gamma_{B}\left(X /{ }_{B} A\right) \vee_{B} \Gamma_{B} A \vee_{B} \Gamma_{B} Y \vee_{B}\left(\Gamma_{B} X\right) \wedge_{B} Y \\
& \simeq{ }_{B} \Gamma_{B}\left(X /{ }_{B} A\right) \vee_{B} \Gamma_{B} A \vee_{B} \Gamma_{B} Y \vee_{B}\left\{\Gamma_{B}\left(X /{ }_{B} A\right) \vee_{B} \Gamma_{B} A\right\} \wedge_{B} Y \\
& \simeq{ }_{B} \Gamma_{B}\left(X /{ }_{B} A\right) \vee_{B} \Gamma_{B} A \vee_{B} \Gamma_{B} Y \vee_{B} \Gamma_{B}\left\{\left(X /{ }_{B} A\right) \wedge{ }_{B} Y\right\} \vee_{B} \Gamma_{B}\left(A \wedge_{B} Y\right) .
\end{aligned}
$$

(2) is obtained similarly.

q.e.d.

Theorem 2.5. Let $\Gamma$ be a fibrewise co-looplike space. Let $(X, A)$ and $(Y, D)$ be fibrewise pointed pairs of fibrewise non-degenerate spaces. Let $\left(X \times{ }_{B} D\right) \cup$ $\left(A \times{ }_{B} Y\right)$ be a subspace of $X \times{ }_{B} Y$.

(1) Let $(X, A)$ be a closed fibrewise cofibred pair. If $A$ is a fibrewise pointed homotopy retraction of $X$, then there is a fibrewise pointed homotopy decomposition

$$
\begin{aligned}
\Gamma_{B}\left\{\left(X \times_{B} D\right) \cup\left(A \times_{B} Y\right)\right\} \simeq & { }_{B} \Gamma_{B} A \vee_{B} \Gamma_{B} Y \vee_{B} \Gamma_{B}\left(A \wedge{ }_{B} Y\right) \\
& \vee_{B} \Gamma_{B}\left(X /{ }_{B} A\right) \vee_{B} \Gamma_{B}\left\{\left(X /{ }_{B} A\right) \wedge_{B} D\right\} .
\end{aligned}
$$

(2) Let $(X, A)$ and $(Y, D)$ be closed fibrewise cofibred pairs. If $A$ is a fibrewise pointed homotopy retraction of $X$ and $D$ is a fibrewise pointed homotopy retraction of $Y$, then there is a fibrewise pointed homotopy decomposition 


$$
\begin{aligned}
\Gamma_{B}\left\{\left(X \times_{B} D\right) \cup\left(A \times_{B} Y\right)\right\} \simeq & { }_{B} \Gamma_{B} A \vee_{B} \Gamma_{B} D \vee_{B} \Gamma_{B}\left(A \wedge{ }_{B} D\right) \\
& \vee_{B} \Gamma_{B}\left(X /{ }_{B} A\right) \vee_{B} \Gamma_{B}\left(Y /{ }_{B} D\right) \\
& \vee_{B} \Gamma_{B}\left\{A \wedge_{B}\left(Y^{*} /{ }_{B} D\right)\right\} \vee_{B} \Gamma_{B}\left\{\left(X /{ }_{B} A\right) \wedge_{B} D\right\} .
\end{aligned}
$$

Proof. (1) Let $M=\left(X \times_{B} D\right) \cup\left(A \times{ }_{B} Y^{*}\right)$. Since $A \times{ }_{B} Y^{*}$ is a fibrewise pointed homotopy retraction of $M$ and $A \times_{B} Y^{\prime} \rightarrow M$ is a fibrewise pointed cofibration by (20.7) and (21.2) of [8], we have

$$
\begin{aligned}
\Gamma_{B} M & \simeq{ }_{B} \Gamma_{B}\left(A \times_{B} Y\right) \vee_{B} \Gamma_{B}\left\{M /{ }_{B}\left(A \times_{B} Y\right)\right\} \\
& \simeq{ }_{B} \Gamma_{B}\left(A \times_{B} Y\right) \vee_{B} \Gamma_{B}\left\{\left(\left(X /{ }_{B} A\right) \times_{B} D\right) /{ }_{B}\left(\left\{*_{A}\right\} \times_{B} D\right)\right\} \\
& \simeq{ }_{B} \Gamma_{B} A \vee_{B} \Gamma_{B} Y \vee_{B} \Gamma_{B}\left(A \wedge_{B} Y\right) \vee_{B} \Gamma_{B}\left(X /{ }_{B} 4\right) \vee_{B} \Gamma_{B}\left\{\left(X /{ }_{B} A\right) \wedge_{B} D\right\}
\end{aligned}
$$

(cf. Proof of Theorem 2.2.)

(2) is obtained similarly.

q.e.d.

In the rest of this section we consider examples of $\Gamma_{B}$-retractile subspaces of a fibrewise product space.

Let $M$ be any subspace of $X \times_{B} Y^{r}$ which contains $X \vee_{B} Y$ and $\bar{\jmath}: X \vee_{B} Y \rightarrow M$ the inclusion map.

Proposition 2.6. Let $\Gamma$ be any fibrewise co-Hopf space. Let $M$ be any subspace of $X \times_{B} Y$ which contains $X \vee_{B} Y$. Then $X \vee_{B} Y$ is $\Gamma_{B}$-retractile in $M$, that $i$, there exists a fibrewise pointed map $\bar{\rho}=\bar{\rho}(\Gamma, X, Y, M): \Gamma_{B} M \rightarrow \Gamma_{B}\left(X \vee_{B} Y\right)$ such that $\bar{\rho} \circ \Gamma_{B} \bar{\jmath} \simeq{ }_{B} 1_{\Gamma_{B}\left(X \backslash{ }_{B} Y\right)}$.

Proof. We define a map $\bar{\rho}=\bar{\rho}(\Gamma, X, I, M): \Gamma_{B} M \rightarrow \Gamma_{B}\left(X \vee_{B} Y^{-}\right)$as follows. Let $p_{1}: X \times_{B} Y \rightarrow X$ and $p_{2}: X \times_{B} Y \rightarrow Y$ be the projections and $i_{1}: X \rightarrow X \vee_{B} Y$ and $i_{2}: Y \rightarrow X \vee{ }_{B} Y$ the inclusion maps. Let $\bar{p}_{1}=p_{1} \mid M: M \rightarrow X$ and $\bar{p}_{2}=p_{2} \mid M: M \rightarrow I^{-}$ be the restrictions of the projections. Then define

$$
\bar{\rho}=\nabla_{Z \cdot B^{\circ}}\left\{\Gamma_{B}\left(i_{1} \circ \bar{p}_{1}\right) \bigvee_{B} \Gamma_{B}\left(i_{2} \circ \bar{p}_{2}\right)\right\} \circ \bar{\gamma}=\Gamma_{B}\left(i_{1} \circ \bar{p}_{1}\right) \dot{+}_{B} \Gamma_{B}\left(i_{2} \circ \bar{p}_{2}\right),
$$

where $Z=\Gamma_{B}\left(X \vee_{B} Y^{\top}\right)$ and $\bar{\gamma}: \Gamma_{B} M \rightarrow \Gamma_{B} M \vee_{B} \Gamma_{B} M$ is the fibrewise co-Hopf structure of $\Gamma_{B} M$ induced by the fibrewise co-Hopf structure $\gamma: \Gamma \rightarrow \Gamma \vee_{B} \Gamma$ of $I$.

We remark that $\Gamma_{B}\left(X \vee_{B} Y^{Y}\right) \cong{ }_{B} \Gamma_{B} X \vee_{B} \Gamma_{B} Y$ (cf. (6.1) of [8]). It follows then that

$$
\begin{aligned}
& \bar{\rho} \circ \Gamma_{B} \bar{\eta}\left|\Gamma_{B} X \times{ }_{B}\left\{*_{B}\right\} \simeq{ }_{B} 1_{\Gamma_{B}\left(Y \vee_{B} Y\right)}\right| \Gamma_{B} X \times_{B}\left\{*_{B}\right\} \text { and } \\
& \bar{\rho} \circ \Gamma_{B}{ }_{B} \bar{\jmath}\left|\left\{*_{B}\right\} \times{ }_{B} \Gamma_{B} Y \simeq_{B} 1_{\Gamma_{B}\left(X \vee_{B} Y\right)}\right|\left\{*_{B}\right\} \times{ }_{B} \Gamma_{B} Y .
\end{aligned}
$$

If the inclusion map $\bar{\jmath}: X \vee_{B} I \rightarrow M$ is a fibrewise pointed cofibration, then there is a long fibrewise pointed cofibration sequence 


$$
X \vee_{B} Y \stackrel{\bar{\jmath}}{\longrightarrow} M \stackrel{\bar{q}}{\longrightarrow} M /{ }_{B}\left(X \vee_{B} Y\right) \stackrel{\bar{\delta}}{\longrightarrow} \Sigma_{B}\left(X \vee_{B} Y\right) \stackrel{\Sigma_{B} \bar{\jmath}}{\longrightarrow} \Sigma_{B} M \longrightarrow \cdots
$$

We have the following result for the map $\bar{o}$ in the above sequence.

Corollary 2.7. $\bar{\delta} \simeq_{B} *_{B}: M /{ }_{B}\left(X \vee_{B} Y\right) \rightarrow \Sigma_{B}\left(X \vee{ }_{B} Y\right)$

Proof. Set $\Gamma=\Sigma=B \times S^{1}$ in Proposition 2.6, then we have a map $\bar{\rho}: \Sigma_{B} M$ $\rightarrow \Sigma_{B}\left(X \vee_{B} Y\right)$ such that $\bar{\rho} \circ \Sigma_{B \bar{\jmath}} \simeq{ }_{B} 1_{\Sigma_{B}\left(X \vee_{B} Y\right)}$. Then we see $\bar{\delta}=1_{\Sigma_{B}\left(X \vee_{B} Y\right)} \circ \bar{\delta}=$ $\left(\bar{\rho} \circ \Gamma_{B} \bar{\jmath}\right) \circ \bar{\delta}=\bar{\rho} \circ\left(\sum_{B} \bar{\jmath} \circ \bar{\delta}\right) \simeq{ }_{B} \bar{\rho} \circ *_{B}=*_{B}$.

q.e.d.

Corollary 2.8. Let $\Gamma$ be a fibrewise co-looplike space. If $\bar{\jmath}: X \vee_{B} Y \subset M$ is a fibrewise pointed cofibration, then the following results hold.

(1) There is a short exact sequence

$$
0 \longrightarrow\left[\Gamma_{B}\left\{M /_{B}\left(X \vee_{B} Y\right)\right\}, Z\right]_{B}^{B} \stackrel{\left(\Gamma_{B} \bar{q}\right)^{*}}{\longrightarrow}\left[\Gamma_{B} M, Z\right]_{B}^{B} \stackrel{\left(\Gamma_{B} \bar{j}\right)^{*}}{\longrightarrow}\left[\Gamma_{B}\left(X \vee_{B} Y\right), Z\right]_{B}^{B} \longrightarrow 0
$$

of algebraic loops and homomorphisms for any space $Z$ in $\mathbf{T o p}_{B}^{B}$.

(2) There is a fibrewise pointed homotopy decomposition

$$
\Gamma_{B} M \simeq_{B} \Gamma_{B} X \vee_{B} \Gamma_{B} Y \vee_{B} \Gamma_{B}\left\{M /{ }_{B}\left(X \vee_{B} Y\right)\right\} .
$$

Proof. Consider the following fibrewise pointed cofibration sequence

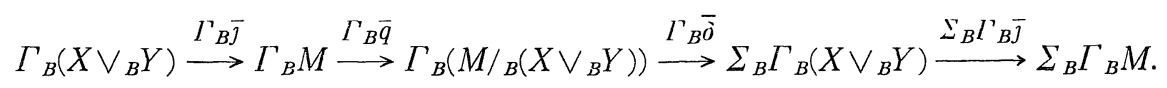

Then we have the result by Proposition 2.6 and Theorem 2.1.

\section{§ 3. $\Gamma_{B}$-Loop Space}

Let $\Gamma$ be a fibrewise co-Hopf space over $B$. For each $X \in \mathbf{T o p}_{B}^{B}$, we define

$$
\Gamma_{L}^{*} X=\operatorname{map}_{B}^{B}(\Gamma, X) \quad\left(\text { the } \Gamma_{B} \text {-loop space of } X\right) \text {. }
$$

A map $f: X \rightarrow Y$ in $\operatorname{Top}_{B}^{B}$ induces a $\Gamma_{B}$ loop map $\Gamma_{B}^{*} f: \Gamma_{B}^{*} X \rightarrow \Gamma_{B}^{*} Y$. We see $\Gamma_{B}^{*} g \circ \Gamma_{B}^{*} f=\Gamma_{B}^{*}(g \circ f)$ for any maps $f: X \rightarrow Y$ and $g: Y \rightarrow Z$ in $\mathbf{T o p}_{B}^{B}$. Let $\gamma: \Gamma \rightarrow$ $\Gamma \vee_{B} \Gamma$ be the fibrewise co-multiplication of $\Gamma$. We define $\gamma_{X}^{*}=\operatorname{map}_{B}^{B}\left(\gamma, 1_{X}\right)$, namely,

$$
\gamma_{X}^{*}: \operatorname{map}_{B}^{B}(\Gamma, X) \times_{B} \operatorname{map}_{B}^{B}(\Gamma, X) \cong{ }_{B} \operatorname{map}_{B}^{B}\left(\Gamma \vee_{B} \Gamma, X\right) \longrightarrow \operatorname{map}_{B}^{B}(\Gamma, X) .
$$

(cf. (9.19) of [8].) Then $\Gamma_{B}^{*} X$ is a fibrewise Hopf space with a fibrewise multiplication $\gamma_{X}^{*}$ for any space $X$ in $\operatorname{Top}_{B}^{B}$. There is a following isomorphism as fibrewise Hopf spaces

$$
\Gamma_{B}^{*}\left(X \times{ }_{B} Y\right) \cong{ }_{B} \Gamma_{B}^{*} X \times{ }_{B} \Gamma_{B}^{*} Y
$$

(cf. (9.9) of [8]). We have formulas 


$$
\Gamma_{B}^{*} \alpha \circ\left(\beta+{ }_{B} \gamma\right)=\left(\Gamma_{B}^{*} \alpha \circ \beta\right)+{ }_{B}\left(I_{B}^{*} \alpha \circ \gamma\right) \text { and }\left(\beta+{ }_{B} \gamma\right) \circ \delta=(\beta \circ \delta)+{ }_{B}(\gamma \circ \delta)
$$

for any maps $\alpha: Y \rightarrow Z, \beta, \gamma: X \rightarrow \Gamma_{B}^{*} Y$ and $\delta: W \rightarrow X$ in $\mathbf{T o p}_{B}^{B}$.

A map $p: E \rightarrow Z$ in $\mathbf{T o p}_{B}^{B}$ is said to be $\Gamma_{b}^{*}$ retractile if $\Gamma_{B}^{*} p: \Gamma_{B}^{*} E \rightarrow I_{b}^{*} Z$ has a fibrewise pointed homotopy cross-section $s: \Gamma_{B}^{*} Z \rightarrow \Gamma_{B}^{*} E$, namely, $\left(\Gamma_{B}^{*} p\right) \circ s$ $\simeq{ }_{B} 1_{\Gamma_{B}^{*} Z}$.

Theorem 3.1. Let $\Gamma$ be a fibrewise co-looplike space. Let $F \stackrel{\imath}{\rightarrow} \stackrel{p}{\rightarrow} Z$ be a fibrewise pointed fibration sequence in $\operatorname{Top}_{B}^{B}$. If $p: E \rightarrow Z$ is $\Gamma_{B}^{*}$-retractile, then the following results hold.

(1) There is a short exact sequence

$$
0 \longrightarrow\left[\Lambda, \Gamma_{B}^{*} F\right]_{B}^{B} \stackrel{\left(\Gamma_{B}^{*} i\right)_{*}}{\longrightarrow}\left[A, \Gamma_{B}^{*} E\right]_{B}^{B} \stackrel{\left(\Gamma_{B}^{*} p\right)_{*}}{\longrightarrow}\left[A, \Gamma_{B}^{*} Z\right]_{B}^{B} \longrightarrow 0
$$

of algebraic loops and homomorphisms for any space $A$ in $\mathbf{T o p}_{B}^{B}$.

(2) There is a fibrewise pointed homotopy decomposition

$$
\Gamma_{B}^{*} E \simeq{ }_{B} \Gamma_{B}^{*} F \times_{B} \Gamma_{B}^{*} Z .
$$

(It does not preserve fibrewise Hopf structure in general.)

Proof. Since $\Gamma_{B}^{*} F \stackrel{\Gamma_{B}^{*}}{\longrightarrow} \Gamma_{B}^{*} E \stackrel{\Gamma_{B}^{*} p}{\longrightarrow} \Gamma_{B}^{*} Z$ is a fibrewise pointed fibration sequence, we have a long fibrewise pointed fibration sequence

$$
\cdots \longrightarrow \Omega_{B} \Gamma_{B}^{*} E \stackrel{\Omega_{B} \Gamma_{B}^{*} p}{\longrightarrow} \Omega_{B} \Gamma_{B}^{*} Z \stackrel{\Gamma_{B}^{*} \delta}{\longrightarrow} \Gamma_{B}^{*} F \stackrel{\Gamma_{B}^{*} i}{\longrightarrow} \Gamma_{B}^{*} E \stackrel{I_{B}^{*} p}{\longrightarrow} \Gamma_{B}^{*} Z
$$

(cf. Crabb and James [2]). Since $\Gamma_{B}^{*} p: \Gamma_{B}^{*} E \rightarrow \Gamma_{B}^{*} Z$ has a fibrewise pointed homotopy cross-section $s: \Gamma_{B}^{*} Z \rightarrow \Gamma_{B}^{*} E$ such that $\left(\Gamma_{B}^{*} p\right) \circ s \simeq_{B} 1_{\Gamma_{B}^{*}} Z$ by our assumption, we have the result by Theorem 1.2 .

q.e.d.

Proposition 3.2. Let $\Gamma$ be a fibrewise co-looplike space. Let $M$ be any subspace of $X \times_{B} Y$ which contains $X \vee_{B} Y$. Let $j: M \rightarrow X \times_{B} Y$ be the inclusion map. Then $\tilde{\eta}$ is $\Gamma_{B}^{*}$-retractile, that is, there exists a fibrewise pointed map $\tilde{\sigma}=$ $\tilde{\sigma}(\Gamma, X, Y, M): \Gamma_{B}^{*}\left(X \times{ }_{B} Y\right) \rightarrow \Gamma^{*} M$ such that $\left(\Gamma_{B}^{*} \tilde{j}\right) \circ \tilde{\sigma} \simeq{ }_{B} 1_{\left.\Gamma_{B}^{*}{ }^{(X \vee}{ }_{B} Y\right)}$.

Proof. Since $\Gamma_{B}^{*} M$ is a fibrewise Hopf space, we can define a map

$$
\tilde{\sigma}=\tilde{\gamma} \circ\left\{\Gamma_{B}^{*}\left(\tilde{l}_{1} \circ p_{1}\right) \times_{B} \Gamma_{B}^{*}\left(\tilde{i}_{2} \circ p_{2}\right)\right\} \circ \Delta_{C, B}=\Gamma_{B}^{*}\left(\tilde{l}_{1} \circ p_{1}\right)+{ }_{B} \Gamma_{B}^{*}\left(\tilde{l}_{2} \circ p_{2}\right)
$$

where $p_{1}: X \times_{B} Y \rightarrow X, p_{2}: X \times_{B} Y \rightarrow Y$ are projections and $\tilde{\imath}_{1}: X \rightarrow M, \tilde{\imath}_{2}: Y \rightarrow M$ are inclusions and $C=\Gamma_{B}^{*}\left(X \times{ }_{B} Y\right)$. The map $\tilde{\gamma}$ is the fibrewise Hopf structure of $\Gamma_{B}^{*} M$ induced by the fibrewise co-Hopf structure $\gamma: \Gamma \rightarrow \Gamma \vee_{B} \Gamma$ of $\Gamma$.

We remark that $\Gamma_{B}^{*}\left(X \times_{B} Y\right) \cong{ }_{B} \Gamma_{B}^{*} X \times_{B} \Gamma_{B}^{*} Y$. Then we have 


$$
\begin{aligned}
\left(\Gamma_{B}^{*} p_{1}\right) \circ\left(\Gamma_{B}^{*} \tilde{j}\right) \circ \tilde{\sigma} & =\Gamma_{B}^{*} p_{1} \circ \Gamma_{B}^{*} \tilde{j} \circ\left\{\Gamma_{B}^{*}\left(\tilde{\imath}_{1} \circ p_{1}\right)+{ }_{B} \Gamma_{B}^{*}\left(\tilde{\imath}_{2} \circ p_{2}\right)\right\} \\
& =\Gamma_{B}^{*}\left(p_{1} \circ \tilde{j}^{\circ} \tilde{\imath}_{1} \circ p_{1}\right)+{ }_{B} \Gamma_{B}^{*}\left(p_{1} \circ \tilde{j}^{\circ} \tilde{\imath}_{2} \circ p_{2}\right) \\
& =\Gamma_{B}^{*} p_{1}+{ }_{B}{ }_{B}{ }_{B}{ }_{B} \Gamma_{B}^{*} p_{1}=\left(\Gamma_{B}^{*} p_{1}\right) \circ 1_{\Gamma_{B}\left(X \times_{B} Y\right)}^{*} .
\end{aligned}
$$

Similarly, we have $\left(\Gamma_{B}^{*} p_{2}\right) \circ\left(\Gamma_{B}^{*} \tilde{j}\right) \circ \tilde{\sigma} \simeq{ }_{B}\left(\Gamma_{B}^{*} p_{2}\right) \circ 1_{\Gamma_{B}^{*}\left(X \wedge_{B} Y^{\prime}\right)}$. Then we have $\left(\Gamma_{B}^{*} \tilde{\jmath}\right) \circ \tilde{\sigma} \simeq_{B} 1_{\Gamma_{B}^{*}\left(X \times{ }_{B} Y\right)}$.

q.e.d.

Let $X_{b_{B}}^{M} Y$ be the fibrewise homotopy fibre of the inclusion map $\tilde{j}: M \rightarrow$ $X \times{ }_{B} Y$ (cf. [2] or $\S 23$ of [8]). Let $i: X{ }_{b_{B}}^{M} Y \rightarrow M$ be the inclusion map.

Theorem 3.3. Let $\Gamma$ be a fibrewise co-looplike space. Let $M$ be any subspace of $X \times_{B} Y$ which contains $X \vee_{B} Y$. Then the following results hold.

(1) There is a short exact sequence

$$
0 \longrightarrow\left[A, \Gamma_{B}^{*}\left(X{ }_{b}^{M} Y\right)\right]_{B}^{B} \stackrel{\left(\Gamma_{B}^{* i}\right)_{*}^{*}}{\longrightarrow}\left[A, \Gamma_{B}^{*} M\right]_{B}^{B} \stackrel{\left(\Gamma_{B}^{*} j\right)_{*}^{*}}{\longrightarrow}\left[A, \Gamma_{B}^{*}\left(X \times_{B} Y\right)\right]_{B}^{B} \longrightarrow 0
$$

of algebraic loops and homomorphisms for any space $A$ in $\mathbf{T o p}_{B}^{B}$.

(2) There is a fibrewise pointed homotopy decomposition

$$
\Gamma_{B}^{*} M \simeq_{B} \Gamma_{B}^{*} X \times_{B} \Gamma_{B}^{*} Y^{\top} \times_{B} \Gamma_{B}^{*}\left(X{ }_{b}^{M} Y\right) .
$$

Proof. By Theorem 3.1 and Proposition 3.2 we have the result.

\section{$\S 4 . \quad \Sigma_{B}$-Retractile and $\Sigma_{B}^{*}$-Retractile}

Let $p: \Sigma_{B} \Omega_{B} X \rightarrow X$ be the fibrewise adjoint of the identity map $1_{\Omega_{B} X}: \Omega_{B} X$ $\rightarrow \Omega_{B} X$. Sunderland [13] called the map $\gamma: X \rightarrow \Sigma_{B} \Omega_{B} X$ in $\mathbb{T o p}_{B}^{B}$ a fibrewise coretraction when it satisfies $p \circ \gamma \simeq_{B} 1_{X}$. He generalized a result of Ganea [3] to the category $\operatorname{Top}_{B}^{P}$, i. e. he showed that there is a bijection between fibrewise pointed homotopy classes of fibrewise coretractions $X \rightarrow \Sigma_{B} \Omega_{B} X$ and fibrewise pointed homotopy classes of fibrewise co-Hopf structures $X \rightarrow X \vee_{B} X$ (cf. Theorem 1.1 of Ganea [3]). We use this result in the proof of the following propositions.

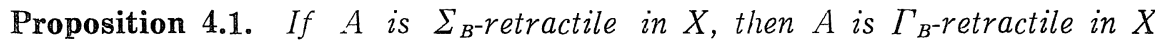
for any fibrewise co-Hopf space $\Gamma$.

Proof. Let $r: \Sigma_{B} X \rightarrow \Sigma_{B} A$ be a fibrewise pointed map such that $r \circ \Sigma_{B} i \simeq_{B} 1_{\Sigma_{B} A}$. Since $\Gamma$ is a fibrewise co-Hopf space, there is a fibrewise pointed space $W\left(=\Omega_{B} \Gamma\right)$ and fibrewise pointed maps $k: \Gamma \rightarrow \Sigma_{B} W$ and $q: \Sigma_{B} W \rightarrow \Gamma$ such that $q \circ k \simeq_{B} 1_{\Gamma}$ by the result of Sunderland [13].

Define $\bar{r}: \Gamma_{B} X \rightarrow \Gamma_{B} A$ by $\bar{r}=\left(q \wedge{ }_{B} 1_{A}\right) \circ\left(1_{W} \wedge_{B} r\right) \circ\left(k \wedge_{B} 1_{X}\right)$ :

$$
\Gamma \wedge_{B} X \longrightarrow \Sigma_{B} W \wedge_{B} X \cong_{B} W \wedge_{B} \Sigma_{B} X \longrightarrow W \wedge_{B} \Sigma_{B} A \cong_{B} \Sigma_{B} W \wedge_{B} A \longrightarrow \Gamma \wedge_{B} A .
$$


Then we have $\bar{r} \circ \Gamma_{B} i \simeq{ }_{B} 1_{\Gamma^{\prime} A}$ by naturality.

q.e.d.

Proposition 4.2. If $p: E \rightarrow Z$ is $\sum_{B}^{*}$-retractile, then it is $\Gamma_{B-}^{*}$-retractile for any fibrewise co-Hopf space $\Gamma$.

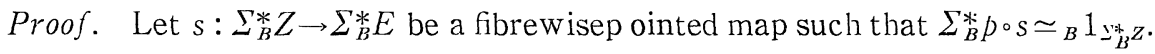
Using the fibrewise pointed maps $k: \Gamma \rightarrow \Sigma_{B} W$ and $q: \Sigma_{B} W \rightarrow \Gamma$ in the proof of Proposition 4.1 , we define $\bar{s}: \Gamma_{B}^{*} Z \rightarrow \Gamma_{b}^{*} E$ by

$$
\bar{s}: \Gamma_{B}^{*} Z \stackrel{q_{B}^{*} Z}{\longrightarrow}\left(\Sigma_{B} W\right)_{B}^{*} Z \cong{ }_{B} W_{B}^{*}\left(\sum_{B}^{*} Z\right) \stackrel{W_{B}^{*} s}{\longrightarrow} W_{B}^{*}\left(\sum_{B}^{*} E\right) \cong{ }_{B}\left(\sum_{B} W\right)_{B}^{*} E \stackrel{k_{B}^{*} E}{\longrightarrow} \Gamma_{B 3}^{*} E .
$$

Then we have $\Gamma_{B}^{*} p \circ S \simeq{ }_{B} 1_{I^{\prime}{ }_{B} Z}$ by naturality. (We used the notation: $\left.X_{B}^{*}\right)^{\prime}=$ $\operatorname{map}_{B}^{B}(X, Y)$.)

q.e.d.

Remark. $\quad \Gamma_{B}$-retractile does not imply $\Sigma_{B^{B}}$-retractile in general. (For example, consider the case $B=\{*\}$ and $\Gamma=\Sigma^{2}$, the double suspension.) But the question of partial converse of Propositions 4.1 and 4.2 , due to Iwase, Sunderland and

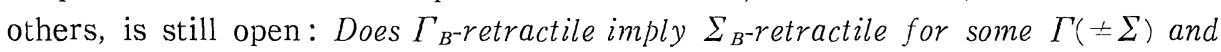
some class of fibrewise pairs $(X,-A)$ ?

\section{References}

[1] Baues, H. J., Algebralc homotopy. Cambridge University Press, Cambridge, 1989.

$2]$ Crabb, M. and James, I. M., Fubrewise homotopy theory, to be published.

[3] Ganea, T., Cogroups and suspensions, Inlent. Math., 9 (1970), 185-197.

[4] Hilton, P., Homotopy theory and dualıty, Notes on Math. and its Appl., Gordon and Breach, New York, London. Paris, 1965.

[5] Hilton, P., Mislin, G. and Roitberg. J., On co-H-spaces, Comm. Math. Helv', 53 (1978), 1-14.

[6] James, I. M., On $H$-spaces and their homotopy groups. Quart. J. Math. Oxford., 11 (1960), 161-179.

โ7」—. General topulogy and humotopy thcory, Springer-Verlag, New York, 1984.

[8] - Fubrewise topoligy. Cambridge University Press, Cambridge, 1989.

[9] Oda, N.. Pairing; of homotopy set; over and under B. Canad, Math. Bull., 36 (1993), 231-240.

[10 $]$ Quillen, D. G., Homotopical algebra, Lect. Note. in Math., 43, Springer-Verlag. Berlin, Hidelberg, New York, 1967.

¿11] Rutter. J. W.. The suspension of the loops on a space with comultiplication. Math. Ann., 209 (1974), 69-82.

[12] Saito. S., Note on co-H-spaces, J. Fac. Sci. Shinshu Univ., 6 (1971), 101-106.

-13] Sunderland, A. M.. Preprint.

[14] Zabrodsky, A., Hopf spaces, North-Holland Math. Stud., 22, North-Holland Publishing Company. Amsterdam. 1976. 
